\title{
NUMERICAL SIMULATIONS OF INTERACTING DISK GALAXIES
}

\author{
Masafumi Noguchi \\ University of Wales, College of Cardiff \\ Cardiff, United Kingdom
}

Galaxy-galaxy interactions have long attracted many extragalactic astronomers in various aspects. A number of computer simulations performed in the 1970s have successfully reproduced the peculiar morphologies observed in interacting disk galaxies and clarified that tidal deformation explains most of the observed global peculiarities. However, most of these simulations have used test particles in modelling the disk component. Tidal response of a self-gravitating disk remains to be further clarified.

Another topic which is intensely discussed at present is the relation between galaxygalaxy interactoins and activity. Many observations suggest that interactions trigger strong starbursts and possibly active galactic nuclei (AGN). However, the detailed mechanism of triggering is not yet clear. It is vital here to understand the dynamics of interstellar gas.

In order to understand various phenomena related to galaxy-galaxy interactions (mainly for disk galaxies), we have performed a series of numerical simulations on close galaxy encounters which includes both interstellar gas and self-gravitating disk components (see Noguchi 1988 for details).

\section{MODELS}

In our simulations, the galaxy model to be perturbed (target galaxy) consists of a halo and a disk. The halo was treated as a rigid spherical gravitational field which is assumed to remain fixed during the interaction. The disk is composed of stars and gas. The stcllar disk was constructed by 20000 collisionless particles of the same mass. Those particles move in the halo gravitational field, interacting with each other and with the perturber. Therefore, the self-gravity of the disk is properly taken into account. Stellar particles were initially given circular velocities with small random motions required to stabilize the disk against local axisymmetric disturbances. The gravitational field of the stellar disk was calculated by the particle-mesh scheme (e.g. Hockney and Eastwood 1981).

The gaseous component was modelled by the cloud-particle scheme (e.g. Roberts and Hausman 1984), namely we represented the gas as an ensemble of small spheres (i.e. clouds) and included the creation of an 'OB star' in a cloud-cloud collision and subsequent velocity push on nearby clouds due to a 'supernova explosion'. Also the cloud-cloud collisions were assumed to be highly inelastic and thus dissipate kinetic energy. The cloud particles are assumed to be massless. Their motion was calculated by using the combined gravitational field of the stellar disk, hale, and perturber. Therefore, the influence of the deformation of the stellar disk in which the gas is embedded was taken into account in addition to the direct effect of the tidal force.

The stability of this galaxy model was confirmed by running the model for a few rotation periods in the absence of a perturbing galaxy. The stellar disk maintains a nearly axisymmetric shape with exponential density distribution, because it is stabilized by a heavy halo component. Only weak, filamentary spiral structures are seen. Also the gas clouds keep moving on nearly circular orbits around the disk center. The velocity dispersion 
of gas clouds stays constant since the energy input by supernova explosions is balanced by dissipation in cloud-cloud collisions.

In the next stage, a point-mass perturber was introduced to disturb this galaxy model (target galaxy). Here we describe only the standard model from the several runs we have carried out. In the standard model, the disk has $20 \%$ of the total target mass (i.e. $80 \%$ is in the halo). The rotation-curve is nearly solid-body from the disk center to about $60 \%$ of the disk radius, outside of which it is nearly flat. The cloud-cloud collision timescale was set to be 1.62 , comparable to the dynamical timescale. One rotation period at the outer disk edge is 6.28 in these units. The perturber has the same mass as the parent galaxy, and moves in a prograde parabolic orbit with perigalactic distance twice the target disk radius.

\section{BAR FORMATION IN THE INNER REGION}

Fig. 1 shows the morphological evolution of the standard model. In the early phase $(T=2.50)$, both stars and gas form a two-armed spiral structure which extends into a tail and bridge, consistent with the results of test-particle simulations (e.g. Toomre and Toomre 1972). The tidal force of the perturber is dominant in this phase.

About one rotation period $\left(6 \times 10^{8} \mathrm{yr}\right.$ for a typical disk galaxy) after the perigalactic passage, a bar structure begins to develop in the inner region of the stellar disk. This point is quite different from the behavior of test-particle disks. In the case of the test-particle disks, only the outer part of the disk is deformed while the inner part remains almost unchanged (e.g. Toomre and Toomre 1972), consistent with the nature of the tidal force. It is evident that the self-gravity of the stellar disk is playing an important role in this bar formation. The initial amplitude of the tidal perturbation given by the perturber is smaller in the inner region than in the outer one. However, disk self-gravity is more dominant in the inner region. The gravitational field in the inner disk region is virtually governed by the disk component itself. The growth of the tidal perturbation is thus greatly accelerated in the inner region. The bar lasts for a few rotational periods but gradually weakens into a slightly oval structure.

Tidally-induced bar formation found here is closely related to the origin of barred galaxies. It is well known that a disk with a mass exceeding that given by the OstrikerPeebles (1973) criterion develops a bar spontaneously. This bar instability may be the generating mechanism of barred galaxies. Comparison of the disk-to-halo mass ratio of barred versus unbarred galaxies would provide a direct check of this hypothesis; the former should be systematically larger than the latter. Unfortunately, the existence of large-scale noncircular motions makes reliable mass estimates difficult for barred galaxies.

The result presented here suggests that close galaxy pairs cam also generate barred galaxies from originally unbarred ones in which the disk mass fraction is small enough that the bar instability did not operate. If this is the case, we should observe barred galaxies more frequently in interacting galaxies than in isolated ones. One possible piece of observational support may come from simple statistics (Noguchi 1987), that spirals in the Atlas of Peculiar Galaxies (Arp 1966) show a slight hint of higher incidence of bars than field spiral galaxies. In the field spirals, $37 \%$ are barred, whereas $55 \%$ of Arp interacting

spirals are barred. Unfortunately the statistical significance is very low in this case. More extensive study is urgently needed. 


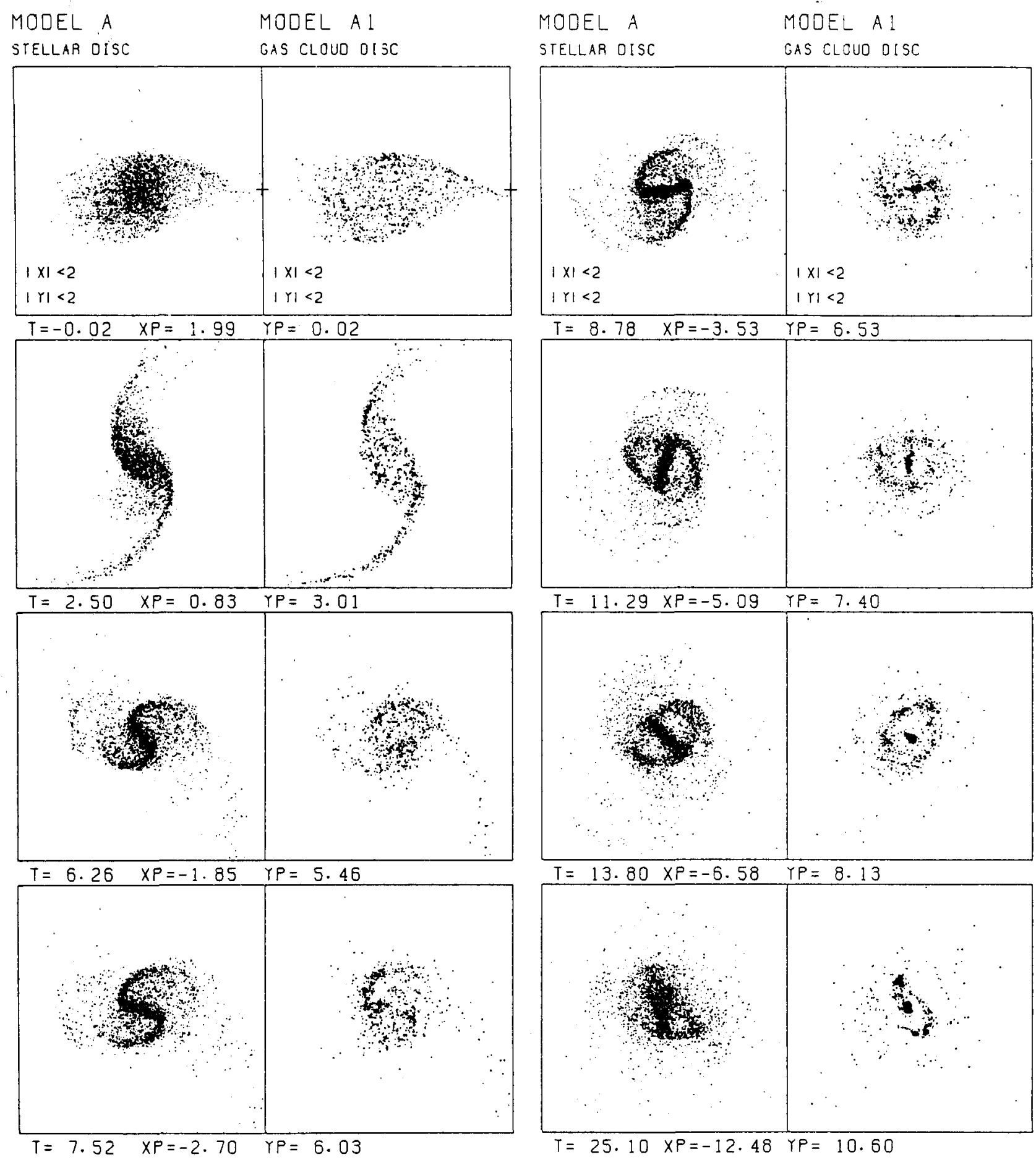

Fig. 1 - A close encounter model of a disk galaxy containing both stars and gas with an equally massive point-mass perturber (from Noguchi 1988). The left and right panels show the stars and gas clouds, respectively. The time $T$ reckoned from perigalactic passage is given in dimensionless units. One unit corresponds to roughly $10^{8} \mathrm{yr}$ for a disk galaxy of typical size and mass. $\mathrm{XP}$ and $\mathrm{YP}$ are $\mathrm{X}$ and $\mathrm{Y}$ coordinates of the perturber, where $\mathrm{X}$ and $\mathrm{Y}$ axes are to the right and upward, respectively. The disk rotates counterclockwise. 


\section{NUCLEAR STARBURSTS FUELLED BY BARS}

The most remarkable observational feature of the starbursts in interacting galaxies is that they take place in the nuclear regions (1-2 kpc) of host galaxies (e.g. Keel et al. 1985). Our numerical models explain the occurrence of nuclear starbursts quite satisfactorily. The right panels in Fig. 1 show the evolution of the gas-cloud disk in the standard model. It is seen that after the stellar bar has developed sufficiently, the gas clouds begin to fall to the disk center. This is because the stellar bar removes the angular momenta and kinetic energy from the gas clouds. About $20 \%$ of the total gas is swallowed within the $10 \%$ radius (1-2 kpc for a typical disk galaxy) from the nucleus up to $T=13$. The maximum infall rate occurs $8-12 \times 10^{8} \mathrm{yr}$ after the perigalactic passage and has a value of about $1 M_{\odot} \mathrm{yr}^{-1}$. Therefore active star formation is expected to take place in the nuclear region about $10^{9}$ yr after the perigalactic passage.

\section{THE CASE OF NGC 1068}

The higher incidence of nuclear starbursts in interacting galaxies seems to be established observationally fairly well. The situation for Seyfert galaxies and other AGN is much more controversial (see the review by Heckman in this volume). However, the well-known Seyfert galaxy NGC 1068 may provide an interesting example. There are several reasons for which we can consider that Seyfert activity in this galaxy has been triggered by a close encounter.

First, NGC 1068 has a possible companion galaxy. Although NGC 1068 is usually treated as an isolated galaxy, it has nearby galaxies such as NGC 1055 and NGC 1073. It is possible that NGC 1068 has interacted with one of these galaxies. Second, NGC 1068 has a stellar bar in the inner region as shown by near-infrared observations (Scoville et al. 1988, Thronson et al. 1989). Myers and Scoville (1987) have found a ring of molecular gas which lies on the ends of the bar, and a sharp peak at the nucleus. The molecular gas distribution they have observed is surprisingly similar to the one shown in Fig. 1 $(T=11.29,13.80)$. Telesco and Decher $(1988)$ have suggested that the bar is located within two inner Lindblad resonances (ILRs). Quite interestingly, a detailed study of individual stellar orbits indicates that the model bar in Fig. 1 is also located within two ILRs. On the other hand, numerical simulations for bar-unstable disks (Miller and Smith 1979, Sellwood 1981) show that the spontaneous bar usually ends near the corotation point. In view of the model results presented here, these observations make NGC 1068 a highly possible case of tidally-triggered Seyfert activity. Detection of a faint H I tidal feature connecting NGC and a companion would further strengthen this picture.

\section{REFERENCES}

Arp, H. 1966, Atlas of Peculiar Galaxies (Pasadena:Caltech).

Hockney, R.W. and Eastwood, J.W. 1981, Computer Simulations Using Particles (New York: McGraw-Hill).

Keel, W.C., Kennicutt, R.C., Jr., van der Hulst, J.M., and Hummel, E. 1985, Astron. J. 90,708 .

Miller, R.H. and Smith, B.F. 1979, Astrophys. J. 227, 785.

Myers, S.T. and Scoville, N.Z. 1987, Astrophys. J. Lett. 312, L39. 
Noguchi, M. 1987, Mon. Not. R. astr. Soc. 228, 635.

Noguchi, M. 1988, Astron. Astrophys. 203, 259.

Ostriker, J.P. and Peebles, P.J.E. 1973, Astrophys. J. 186, 467.

Roberts, W.W. and Hausman, M.A. 1984, Astrophys. J. 277, 744.

Scoville, N.Z., Matthews, K., Carico, D.P. and Sanders, D.B. 1988, Astrophys. J. Lett. $327, \mathrm{~L} 61$.

Sellwood, J.A. 1981, Astron. Astrophys. 99, 362.

Telesco, C.M. and Decher, R. 1988, Astrophys. J. 334, 573.

Thronson. H.A. et al. 1989, Astrophys. J. 343, 158.

Toomre, A. and Toomre, J. 1972, Astrophys. J. 178, 623.

\section{DISCUSSION}

Balsara: I am somewhat uncomfortable with your having a set of simple algebraic rules for making $O B$ stars with the net result of these $O B$ stars being to blow up and heat the disk. So, all you have is an algebraic set of rules for heating the disk. Also, this heating is expected to blow out in the vertical direction (superbubbles and all that) and the old star population is expected to remain unaffected by that.

Noguchi: In the model presented here, the energy input from the explosion of $O B$ stars is negligible compared with the total kinetic energy of stars and gas. In particular, the heating of stellar disk is a purely stellar-dynamical one, and $O B$ stars do not contribute to it. So, the global phenomena discussed here (i.e., the formation of a stellar bar and the inflow of the gas) will not be sensitive to the detailed specification of the star formation process. Heating and blow out due to intensive star formation will be important in the case of small galactic mass.

Sotnikova: Did you consider the interaction between clouds escaping from the parent galaxy with a hot intergalactic medium, which also can lead to the disappearance of clouds.

\section{Noguchi: No}

M. Klaric: What happens with the clouds after they collide?

Noguchi: The clouds create an "OB star" at their center of mass. At the end of its lifetime ( $\left.10^{7} \mathrm{yr}\right)$, the "OB star" explodes as a "supernova" and gives a velocity boost to the clouds which reside within a certain radius (which corresponds to the size of super nova remnant) from the "OB star". 
Zasov: Let's compare two types of galaxies. The first one is that of the early-type spiral, say, Sa - Sbc. The second one is, say, Sc. For the first type of galaxy we have a large non-disk component, large angular velocity, and probably non-self gravitating disk. For the second one, it is the other way around: a self-gravitating (at least in the inner parts) disk and a lower angular rotation. Judging from the results of your numerical modeling, we should expect more violent activity for the late-type galaxies. Nevertheless seyfert nuclei and nuclear hot spots usually occur in early type systems. How can that be made to agree with your results?

Noguchi: It is not clear how the disk mass fraction changes with respect to the spheroidal component (not just the bulge, but the bulge + halo) and hence how the importance of self-gravity changes with the Hubble type. So we should be careful in comparing the results with the observations. Although seyfert galaxies are more common in early type galaxies, nuclear starbursts are more often observed in late type galaxies.

Navarro: When does star formation occur in your simulations, and how sensitive are your results to this?

Noguchi: I assumed that one star formation event takes place per each cloud-cloud collision. I didn't investigate other cases.

Simkin: In the later epochs you see an alternating surge of star formation - first on one side, then on the other side of the bar. Is this sensitive to initial conditions?

Noguchi: I think that this is caused because the tidal interaction deviates from exact bi-symmetry.

Chatterjee: The formation of structures in your simulations seem to be extremely dependent on the rotation velocity of the victim disk?

Noguchi: Yes. For example, the length of the induced bar is well correlated with the radius of the solid-body part of the rotation curve. 